\title{
Evaluation of Indoor Position Localization System by Angle of Arrival Using RSS Based Distance Measurements
}

\author{
Mahmoud F. Mosleh ${ }^{1}$, Ali H. Hashim² ${ }^{2}$ Mohammed J. Zaiter ${ }^{3}$ \\ \{drmahmoodfarhan@gmail.com ${ }^{1}$, ali.hussain.moi2@gmail.com ${ }^{2}$, mjzaiter@yahoo.com ${ }^{3}$ \} \\ Electrical Engineering Technical College, Middle Technical University, Baghdad, Iraq ${ }^{123}$
}

\begin{abstract}
Indoor localization is a technique utilized to estimate the locations of objects and persons tracking. Time of Arrival (ToA) and Recieved Signal Strength (RSS) are the most too important techniques use for such tasks according to its easy implantation and acceptable accuracy. In this paper, AoA is utilized to estimate targets that are deployed in a selected case study building. Wireless InSite (WI) packages are utilized to simulate the case study model and apply our experiment. ToA and RSS are used to estimate distances between the transmitter and each target, then AoA is applied to locate each target. The results show that the errors between actual and estimated distance are in the range of $(0$ $1)$ and $(0.07-7.4)$ for ToA and RSS respectively. The achieved results show that AoA is an effective technique for indoor localization.
\end{abstract}

Keyword - AoA; ToA; RSS; Indoor localization; Wireless InSite.

\section{Introduction}

Modern developments in electronic technology and communication have resulted in the emergence of new applications in different fields including wireless communication with prospective applications. The most applications included environmental monitoring, military services, objective tracking, patient monitoring and tracking [1]. Location-based services (LBS) involve the localization process of finding the target position [2]. In many applications of wireless communication, including tracking and monitoring, the collected data is still not very much useful without knowing the corresponding target positions. In recent years the Indoor Positioning System (IPS) utilizing the radio signal has developed rapidly due to increasing demands for indoor Location-Based-Devices (LSB) [3], [4].

Localization techniques can be divided into range-based and range-free schemes. The first one is widely used due to its high accuracy, ease of implementation and applicability to different radio technologies. Range (distance) estimated from different features of the received radio signal, like time of arrival (ToA), time of flight (ToF), time-difference of arrival (TDoA) and received signal strength (RSS) [5].

RSS is a method to estimate the position location of a target, the distance between two points can be estimated by measuring the energy of the received signal at one end. Distance calculation based on RSS measurements requires the knowledge of channel characteristics. The RSS can be classified to a constant and a variable component. The constant component is affected by a path loss propagation model, and the variable component is affected by many factors include multipath effects, signal attenuation and shadowing. RSS method is highly accurate in short-range and Line-of-Sight (LoS) environment. In addition, it's easier, cheaper 
and does not require time synchronization. However, the RSS method requires at least three reference points to locate the position of a target. Moreover, the power control used in measurements seriously affects the accuracy and can lead to incorrect target positioning. Also, this method does not work perfectly in Non-Line-of -Sight (NLOS) state because of multipath effects [6].

The second important technique for localization positioning is range free which includes distance-vector hop, fingerprint matching and Angle of Arrival AoA measurement [7]. AoA is an example of range free which is represents the angle between an incident wave propagation direction and some reference direction which is called orientation. Orientation, defined as a fixed direction against which the AoA are measured, is represented in degrees in a clockwise direction. AoA determines the direction based on the measurement of TDoA at individual elements of the array, from these delays the AoA can be estimated.

There are many challenges facing indoor localization such as huge and large noise and multipath from other wireless communication like cellular system communication [8]. Inaccuracies in measurement of AoA can be related to the wireless communication channel, the measuring device/method, or both. The spatial features of the wireless channel have a significant effect on the AoA detection [9].

Many researchers reviewed the subject of indoor localization using many techniques such as [8] who present an evaluation of the performance of received power level versus the separation distance between the access points to every received point in four selected locations. The results showed a significant variation from the four determined locations, this variation is related to reflection of different obstacles in indoor environment and different materials effects on signal wave propagation. In [10] where AoAs were estimated by finding the mean received power and determine the mean time of arrival (ToA) to obtain orientation and theta for butter localization in an antenna array. Also, [11] proposed an AoA-based localization with two known access point $4800 \mathrm{Wi}$-Fi with $2.4 \mathrm{GHz}$ to estimate the location for 10 unknown points were used for more accurate result and the localization error was less than 2.5 meter, about $80 \%$ of the time as the result showed in the Line of Sight (LoS) dominating environments. In [2] researchers present an approach for localization using the RSSI values. The method was applied in a 5 x 4 $\mathrm{m}$ indoor environment an average two dimensions localization error of $30 \mathrm{~cm}$ is obtained. In this paper, one building of Electrical Engineering Technical College is selected as a case study to apply an experiment position location for many objects represented by the receiver device (RXs). Also, the number of transmitter device (TXs) is installed in a preplanned location in order to obtain AoA measurement and RSS estimation.

\section{AoA Based Position Localization}

In wireless communication system the AoA estimated the position location in 3D, to calculate the distance between the source and the target according to the speed of electromagnetic wave propagation in indoor environment by mean time of arrival, the distance $\mathrm{d}$ is obtained by:

$$
\mathrm{d}=\mathrm{c} \times \mathrm{t}
$$

Where $\mathrm{c}$ is speed of light $\left(3 \times 10^{8}\right) \mathrm{ms}^{-1}, \mathrm{t}$ is the propagation time in one way signal with maximum power received [10]. 
The source and target separation distance can be utilized to determine the coordinates of the location of target which is represented as target. Such location will be determined with respect of source location and bearing angle $\beta$ between source and target which represented by the relation:

$$
\beta=\alpha+\pi .
$$

Where $\alpha$ is direction of arrival in (phi) calculated by Wireless InSite (WI) package as a position coordination of target (XRX, YRX) which can be estimated by:

$$
\begin{aligned}
& \mathrm{X}_{\mathrm{RX}}=\mathrm{X}_{\mathrm{TX}}+\mathrm{d} \times \cos (\beta) \\
& \mathrm{Y}_{\mathrm{RX}}=\mathrm{Y}_{\mathrm{TX}}+\mathrm{d} \times \sin (\beta)
\end{aligned}
$$

Where $\left(\mathrm{X}_{\mathrm{TX}}, \mathrm{Y}_{\mathrm{TX}}\right)$ represented the TXs known location, equations (3) and (4) are clear out in Figure 1 [12].

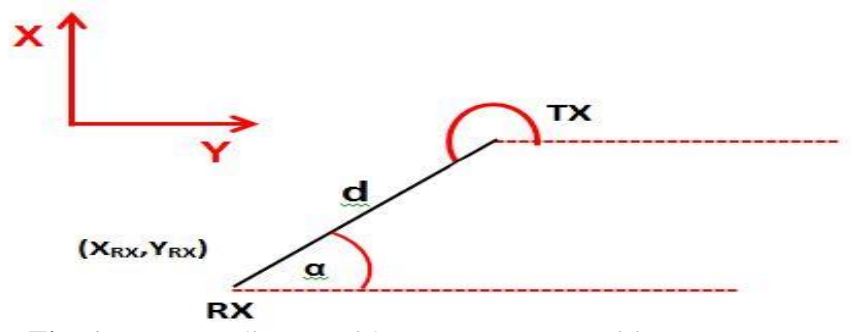

Fig. 1. RX coordinates with respect to TX position.

\section{RSS based distance measurement}

RSS is the strength of a received signal measured at the receiver's antenna. RSS is determined by the transmission power, the distance between the transmitter and the receiver, and the radio environment. All wave signals suffer from phenomena called free-space loss [13] in which the signal becomes weaker as a function of distance squared when propagates in space. The received power can be calculated according to Friis equation:

$$
P_{r}=\frac{P_{t} G_{r} G_{t} \lambda^{2}}{(4 \pi)^{2} d^{2}}
$$


Where $P_{t}$ is the transmitted power, $G_{t}$ and $G_{\mathrm{r}}$ are the antenna gain of the transmitter and receiver respectively, $\lambda$ is the carrier wavelength it is equal to:

$$
\lambda=\frac{\mathrm{f}}{\mathrm{c}}
$$

Where $\lambda$ is the wavelength of the signal and $f$ is the carrier frequency of the signal.

The propagated signals are subjected to various types of attenuations called path loss parameter which can be calculated by [14], [18]:

$$
\text { FSPL }=20 \log _{10}(d)+20 \log _{10}(f)+20 \log _{10}\left(\frac{4 \pi}{c}\right)-G_{t}-G_{r}
$$

Where $\mathrm{d}$ is the distance between transmitter and receiver.

To estimate the distance between the transmitter and receiver coordinator can be based on equation [19]:

$$
\mathrm{d}=10^{\left.-\left(\mathrm{RSS}-\mathrm{P}_{\mathrm{t}}+\mathrm{Pl}_{0}-\sigma\right) / 10 \gamma\right)}
$$

Where $\mathrm{Pl}_{0}$ (FSPL) is the path loss at a reference distance do (1 meter) and by applying equation (7) found to be equal to $(32.64 \mathrm{~dB})$, the path loss exponent $\gamma$ depends on the environment and which is in a range of $(2-6)$, according to [20]. In this paper, $\gamma$ is considered as (4). Also, according to [20], [21], the standard deviation $\sigma$ depended on the environments of case study in the range of $(2-14)$ which assumed to be (9) in this paper.

\section{Case Study}

The 1'st floor of case study building, with a dimension of $(47.5 \mathrm{~m} \times 43.5 \mathrm{~m} \times 3.5 \mathrm{~m})$ is chosen to apply our experiment. Firstly, the simulated building is designed using WI packages and loaded by specific instructions. The resultant building is illustrated in Figure 2(a). Three different locations of TXs device with a height of $2 \mathrm{~m}$ above the floor are set up in various locations highlighted by black color. On the other hand, twenty RXs with $1.5 \mathrm{~m}$ above floor are distributed randomly on the same floor of the building shown as red color in Figure 2(b). Also, all properties of both TX and RX are listed in Table 1.

Additionally, the impact of different building materials (Concrete, Glass, Wood and Brick) on wave propagation with a frequency of $2.4 \mathrm{GHz}$ band and $1 \mathrm{MHz}$ bandwidth was taken into consideration for the entire investigation. Each material thickness, conductivity $(\sigma)$ and relative permittivity $\left(\eta^{\prime}\right)$ was determined based on the recommendation of the International Telecommunication Union (ITU). The results of $\left(\sigma \& \eta^{\prime}\right)$ calculations at a frequency of $2.4 \mathrm{GHz}$ are listed in Table 2 [22].

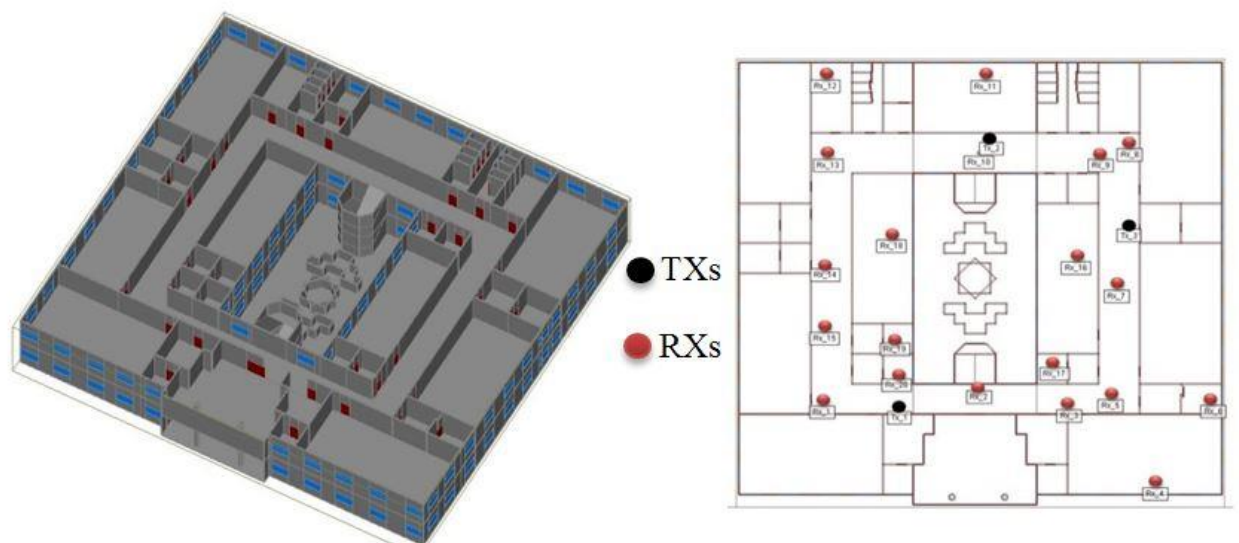


Fig. 2. Simulation model of case study building (a) 3D view (b) TXs and RXs locations.

Table 1. TX and RX antenna properties.

\begin{tabular}{lll}
\hline Antenna properties & TX Antenna & RX Antenna \\
\hline Antenna type & Omni-Directional & Omni-Directional \\
Input Power $(\mathrm{dBm})$ & 6 & - \\
Gain $(\mathrm{dBi})$ & 5.4 & 2 \\
Waveform & Sinusoid & Sinusoid \\
Polarization & $\mathrm{V}$ & $\mathrm{V}$ \\
(VSWR) & 1 & 1 \\
\hline Where (VSWR) is the Value for the antenna's voltage standing wave ratio.
\end{tabular}

Table 2. Material Thickness, Conductivity and Permitivity values.

\begin{tabular}{cccc}
\hline Materials & Thickness $(\mathrm{m})$ & $\sigma$ & $\eta$ \\
\hline Concrete & 0.125 & 0.066 & 5.31 \\
Glass & 0.003 & 0.012 & 6.27 \\
Wood & 0.030 & 0.012 & 1.99 \\
Brick & 0.125 & 0.038 & 3.75 \\
\hline
\end{tabular}




\section{Results and Discussion}

As with previous explanation of sections 2 and 3, the location can be estimated by only one TX when depending on equations (3) and (4), but for more accuracy, three TXs are deployed. The estimated distance using Wireless InSite (WI) package based on mean time of arrival, while the estimated distance based on RSS values obtained according to equation (8). The RSS values determined using Wi-Fi meter application. There was a slight difference in distance measurement in both ways, this difference can be explained by the fact that the RSS measurement affected by multipath effects, signal attenuation and shadowing. In addition to indoor environment infrastructure for example (Objects, Furniture, Forner, Other electrical and electronic devices, etc), this difference can be noticed in Figure 3. which illustrates the estimated distance based on mean ToA and RSS simultaneously.

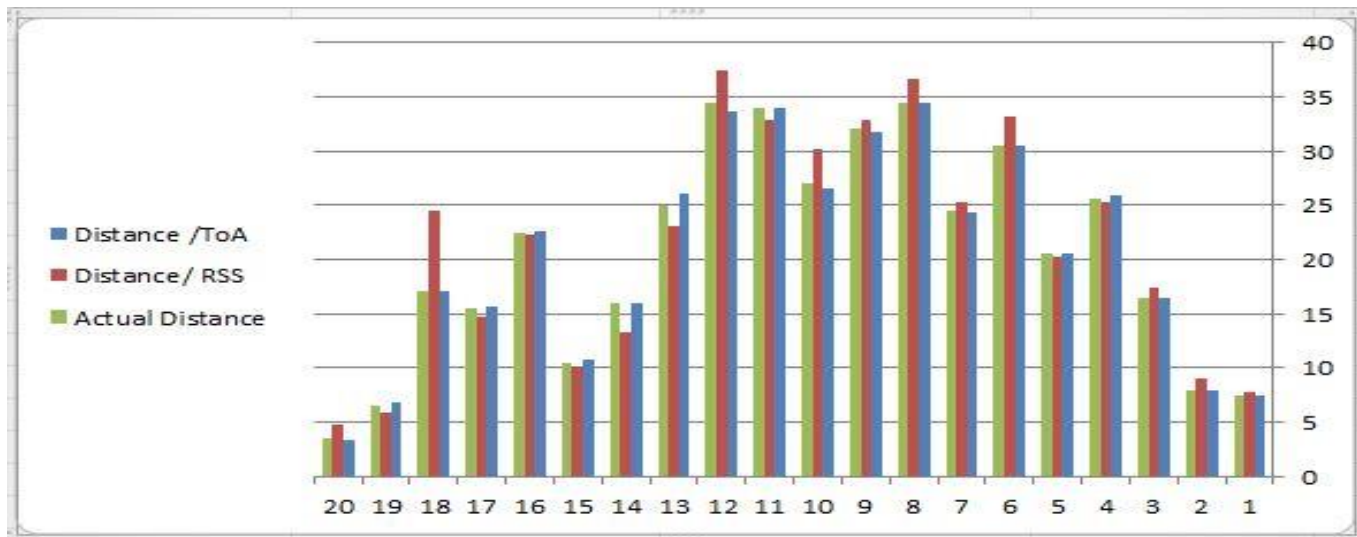

(a)

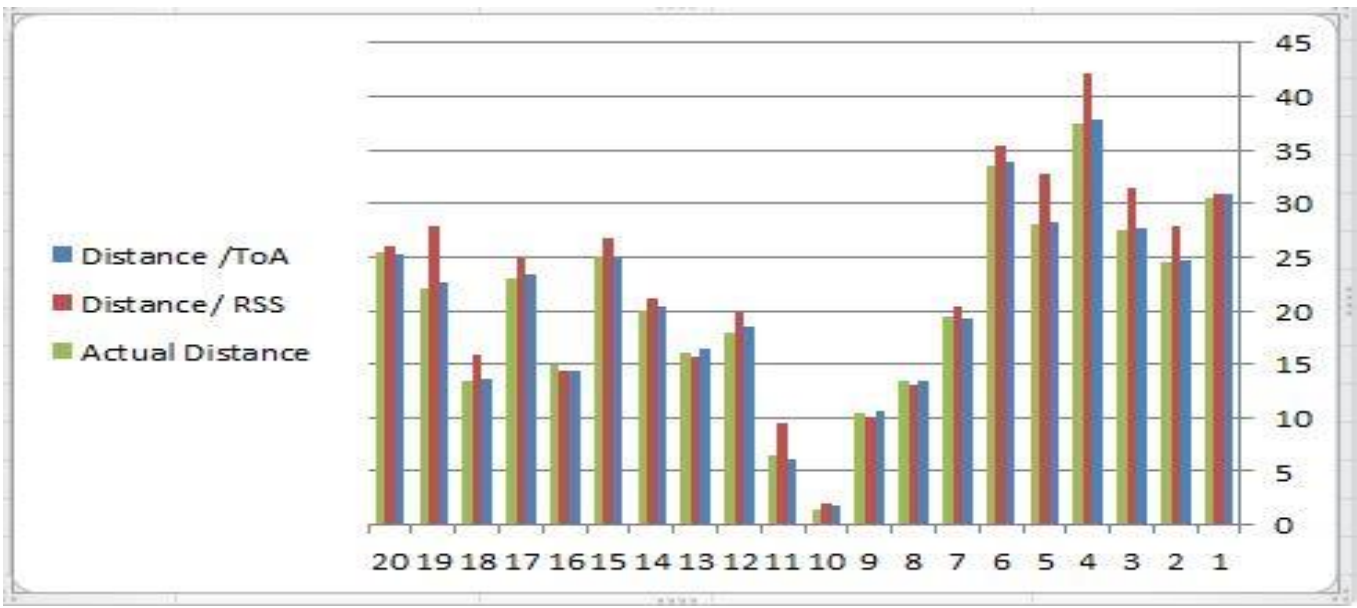


(b)

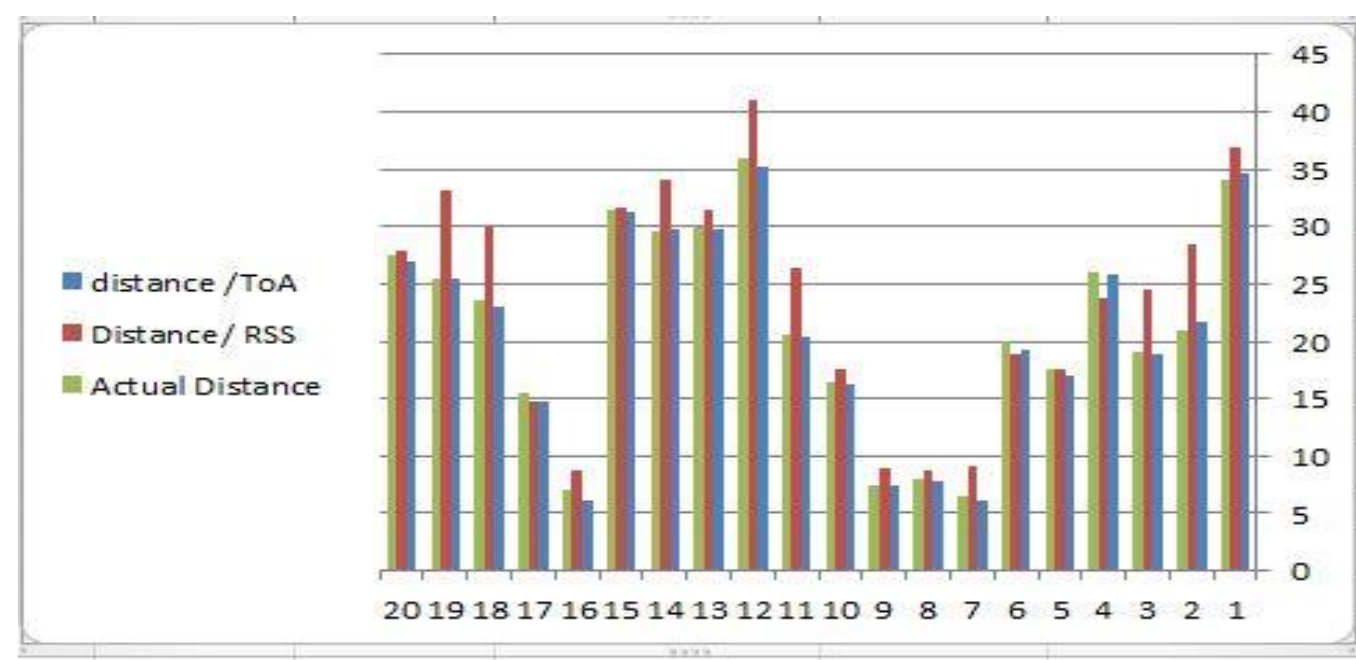

(c)

Fig. 3. Estimated distance based on Mean ToA and RSS in:

(a) TX-1, (b) TX-2 and (c) TX-3.

As illustrated in Table 3. The RSS based distance error was estimated and maximum error value found to be $(7.4,5.9,7.7)$ meter in TX-1, TX-2 and TX-3 respectively These slight errors in distance estimation is due to indoor environment infrastructure. On the other hand, the maximum value for ToA based distance error was $(1,0.6,0.9)$ meter in TX-1, TX-2 and TX-3 respectively.

Table 3. Showed the estimated distance based on mean ToA and RSS with distance error for (a) TX-1,

(b) TX-2 and (c) TX-3.

\begin{tabular}{cccccccc}
\hline Tx-1 & location & $\begin{array}{c}(15.5,8.5) \\
\text { Distance } \\
\text { RoA }\end{array}$ & Pr & Distance/RSS & $\begin{array}{c}\text { Actual } \\
\text { Distance }\end{array}$ & $\begin{array}{c}\text { Distance Errors } \\
\text { /ToA }\end{array}$ & $\begin{array}{c}\text { Distance Errors } \\
\text { /RSS }\end{array}$ \\
\hline Rx_1 & 355.74 & 7.5 & -53.3 & 7.7893 & 7.5 & 0 & 0.289 \\
Rx_2 & 194.88 & 7.9 & -55.7 & 8.9433 & 7.9 & 0 & 1.043 \\
Rx_3 & 180.59 & 16.5 & -67.2 & 17.338 & 16.5 & 0 & 0.838 \\
Rx_4 & 163 & 25.9 & -73.7 & 25.206 & 25.6 & 0.3 & 0.394
\end{tabular}




\begin{tabular}{lccccccc} 
Rx_5 & 183.29 & 20.5 & -69.9 & 20.253 & 20.5 & 0 & 0.247 \\
Rx_6 & 181.38 & 30.5 & -78.5 & 33.228 & 30.5 & 0 & 2.728 \\
Rx_7 & 211.47 & 24.3 & -73.8 & 25.351 & 24.5 & 0.2 & 0.851 \\
Rx_8 & 229.83 & 34.4 & -80.2 & 36.644 & 34.5 & 0.1 & 2.144 \\
Rx_9 & 233.94 & 31.8 & -78.3 & 32.847 & 32 & 0.2 & 0.847 \\
Rx_10 & 253.66 & 26.5 & -76.8 & 30.13 & 27 & 0.5 & 3.13 \\
Rx_11 & 256.53 & 33.9 & -78.3 & 32.847 & 34 & 0.1 & 1.153 \\
Rx_12 & 282.68 & 33.7 & -80.6 & 37.497 & 34.5 & 0.8 & 2.997 \\
Rx_13 & 285.48 & 26 & -72.2 & 23.121 & 25 & 1 & 1.879 \\
Rx_14 & 297.43 & 15.9 & -62.5 & 13.228 & 16 & 0.1 & 2.772 \\
Rx_15 & 313.2 & 10.7 & -57.8 & 10.093 & 10.5 & 0.2 & 0.407 \\
Rx_16 & 221.35 & 22.6 & -71.6 & 22.336 & 22.5 & 0.1 & 0.164 \\
Rx_17 & 197.15 & 15.6 & -64.3 & 14.672 & 15.5 & 0.1 & 0.828 \\
Rx_18 & 272.83 & 17.1 & -73.2 & 24.491 & 17 & 0.1 & 7.491 \\
Rx_19 & 274.46 & 6.8 & -48.5 & 5.9088 & 6.5 & 0.3 & 0.591 \\
Rx_20 & 276.12 & 3.3 & -44.5 & 4.6935 & 3.5 & 0.2 & 1.194 \\
\hline
\end{tabular}

(a)

\begin{tabular}{|c|c|c|c|c|c|c|c|}
\hline $\begin{array}{c}\text { Tx-2 } \\
\text { Receives }\end{array}$ & $\begin{array}{l}\text { Location } \\
\qquad \alpha\end{array}$ & $\begin{array}{c}(24.5,35.5) \\
\text { Distance /ToA }\end{array}$ & $\operatorname{Pr}$ & Distance/ RSS & $\begin{array}{c}\text { Actual } \\
\text { Distance } \\
\end{array}$ & $\begin{array}{c}\text { Distance Errors } \\
\text { /ToA }\end{array}$ & $\begin{array}{c}\text { Distance Errors } \\
\text { /RSS } \\
\end{array}$ \\
\hline$R x \_1$ & 57.75 & 30.9 & -77 & 30.832 & 31 & 0.4 & 0.3318795 \\
\hline$R x \_2$ & 91.86 & 24.74 & -75 & 27.797 & 25 & 0.24 & 3.29713268 \\
\hline $\mathrm{Rx} \_3$ & 106.32 & 27.64 & -78 & 31.369 & 28 & 0.14 & 3.8689513 \\
\hline $\mathrm{Rx} \_4$ & 114.99 & 37.8 & -83 & 42.073 & 38 & 0.3 & 4.57266284 \\
\hline $\mathrm{Rx} \_5$ & 114.16 & 28.2 & -78 & 32.847 & 28 & 0.2 & 4.84732443 \\
\hline Rx_6 & 131.93 & 33.9 & -80 & 35.4 & 34 & 0.4 & 1.89973411 \\
\hline $\mathrm{Rx} \_7$ & 130.44 & 19.3 & -70 & 20.37 & 20 & 0.2 & 0.87042078 \\
\hline $\mathrm{Rx} \_8$ & 177.4 & 13.4 & -62 & 13.152 & 14 & 0.1 & 0.34775168 \\
\hline Rx_9 & 171.01 & 10.7 & -58 & 10.151 & 11 & 0.2 & 0.34920633 \\
\hline $\mathrm{Rx} \_10$ & 47.57 & 1.8 & -30 & 2.0725 & 1.5 & 0.3 & 0.57252606 \\
\hline $\mathrm{Rx} \_11$ & 275.28 & 6.2 & -57 & 9.528 & 6.5 & 0.3 & 3.02796164 \\
\hline$R x \_12$ & 30.75 & 18.5 & -70 & 19.792 & 18 & 0.5 & 1.79247021 \\
\hline$R x \_13$ & 5.94 & 16.5 & -66 & 15.722 & 16 & 0.5 & 0.27828208 \\
\hline$R x \_14$ & 37.62 & 20.4 & -71 & 21.208 & 20 & 0.4 & 1.20801393 \\
\hline $\mathrm{Rx} \_15$ & 50 & 24.9 & -75 & 26.853 & 25 & 0.1 & 1.85344446 \\
\hline$R x \_16$ & 125.28 & 14.4 & -64 & 14.338 & 15 & 0.6 & 0.66162284 \\
\hline
\end{tabular}




\begin{tabular}{lccccccc} 
Rx_17 & 108.29 & 23.3 & -74 & 24.917 & 23 & 0.3 & 1.91724371 \\
Rx_18 & 45.13 & 13.7 & -66 & 15.904 & 14 & 0.2 & 2.40376684 \\
Rx_19 & 66.46 & 22.6 & -76 & 27.958 & 22 & 0.6 & 5.95760728 \\
Rx_20 & 69.5 & 25.2 & -74 & 25.942 & 26 & 0.3 & 0.44179362 \\
\hline
\end{tabular}

(b)

\begin{tabular}{|c|c|c|c|c|c|c|c|}
\hline $\begin{array}{c}\text { Tx-3 } \\
\text { Receives }\end{array}$ & $\begin{array}{c}\text { location } \\
\alpha\end{array}$ & $\begin{array}{c}(38,26.5) \\
\text { distance } / \text { ToA }\end{array}$ & $\mathrm{pr}$ & Distance/ RSS & $\begin{array}{c}\text { Actual } \\
\text { Distance }\end{array}$ & $\begin{array}{c}\text { Distance Errors } \\
\text { /ToA }\end{array}$ & $\begin{array}{c}\text { Distance Errors } \\
\text { /RSS }\end{array}$ \\
\hline $\mathrm{Rx} \_1$ & 31.46 & 34.6 & -80 & 36.8553 & 34 & 0.6 & 2.8553 \\
\hline $\mathrm{Rx} \_2$ & 54.2 & 21.7 & -76 & 28.44461 & 21 & 0.7 & 7.44461 \\
\hline $\mathrm{Rx} \_3$ & 71.6 & 18.8 & -73 & 24.49063 & 19 & 0.2 & 5.49063 \\
\hline Rx_4 & 95.59 & 25.8 & -73 & 23.79578 & 26 & 0.2 & 2.20422 \\
\hline $\mathrm{Rx} \_5$ & 83.87 & 17 & -68 & 17.64006 & 17.5 & 0.5 & 0.14006 \\
\hline Rx_6 & 114.5 & 19.3 & -69 & 18.90166 & 20 & 0.7 & 1.09834 \\
\hline Rx_7 & 78.77 & 6.1 & -56 & 9.204496 & 6.5 & 0.4 & 2.7045 \\
\hline $\mathrm{Rx} \_8$ & 269.6 & 7.8 & -55 & 8.73977 & 8 & 0.2 & 0.73977 \\
\hline Rx_9 & 291.6 & 7.4 & -56 & 8.994976 & 7.5 & 0.1 & 1.49498 \\
\hline $\mathrm{Rx} \_10$ & 334.3 & 16.3 & -67 & 17.53881 & 16.5 & 0.2 & 1.03881 \\
\hline $\mathrm{Rx} \_11$ & 313.8 & 20.3 & -75 & 26.39368 & 20.5 & 0.2 & 5.89368 \\
\hline $\mathrm{Rx} \_12$ & 25.03 & 35.2 & -82 & 41.11497 & 36 & 0.8 & 5.11497 \\
\hline $\mathrm{Rx} \_13$ & 346.7 & 29.7 & -78 & 31.36895 & 30 & 0.3 & 1.36895 \\
\hline $\mathrm{Rx} \_14$ & 7.42 & 29.8 & -79 & 34.00165 & 29.5 & 0.3 & 4.50165 \\
\hline$R x \_15$ & 21.34 & 31.3 & -78 & 31.73219 & 31.5 & 0.2 & 0.23219 \\
\hline $\mathrm{Rx} \_16$ & 32.14 & 6.1 & -55 & 8.73977 & 7 & 0.9 & 1.73977 \\
\hline$R x \_17$ & 61.77 & 14.8 & -65 & 14.84226 & 15.5 & 0.7 & 0.65774 \\
\hline $\mathrm{Rx} \_18$ & 4.41 & 23.1 & -77 & 29.95712 & 23.5 & 0.4 & 6.45712 \\
\hline$R x \_19$ & 27.12 & 25.4 & -79 & 33.22768 & 25.5 & 0.1 & 7.72768 \\
\hline $\mathrm{Rx} \_20$ & 34.79 & 27 & -76 & 27.95761 & 27.5 & 0.5 & 0.45761 \\
\hline
\end{tabular}

(c)

In Table 4, ToA and RSS based distance utilized to determine each receiver coordinates using equation (3) and (4), and the results show that ToA based AoA location determination was slightly more accurate than RSS based method. 
Table 4. Position localization ToA and RSS based AoA estimated.

\begin{tabular}{|c|c|c|c|c|c|c|c|c|c|c|}
\hline \multirow[b]{2}{*}{ Receive } & \multicolumn{2}{|c|}{$\begin{array}{c}\text { location } \\
\text { Estimated Location AoA } \\
\text { /ToA }\end{array}$} & $\begin{array}{l}\text { Estim } \\
\text { A }\end{array}$ & $\begin{array}{l}\text { Location } \\
\text { RSS }\end{array}$ & & $\begin{array}{l}\text { ual } \\
\text { tion }\end{array}$ & \multirow{2}{*}{$\begin{array}{c}\text { Errors AoA / } \\
\text { ToA } \\
\text { X }\end{array}$} & \multicolumn{3}{|c|}{$\begin{array}{c}\text { Errors AoA / } \\
\text { RSS }\end{array}$} \\
\hline & $\mathrm{X}$ & Y & $\mathrm{X}$ & $\mathrm{Y}$ & $\mathrm{X}$ & $\mathrm{Y}$ & & $\mathrm{Y}$ & $\mathrm{X}$ & Y \\
\hline$R x \_1$ & 8.02 & 9.06 & 7.8 & 9.07 & 8 & 9 & 0.02 & 0 & 0 & 0 \\
\hline$R x \_2$ & 23.1 & 10.51 & 23.13 & 10.51 & 24 & 10.5 & 0.4 & 0 & 0 & 0 \\
\hline $\mathrm{Rx} \_3$ & 32 & 8.64 & 31.7 & 8.64 & 32 & 8.7 & 0.2 & 0 & 1 & 0 \\
\hline Rx_4 & 40.2 & 0.92 & 39.5 & 1.13 & 41 & 1 & 0.3 & 0 & 1 & 0 \\
\hline $\mathrm{Rx} \_5$ & 35.9 & 9.67 & 35.6 & 9.62 & 36 & 9.75 & 0.1 & 0 & 0 & 0 \\
\hline Rx_6 & 45.9 & 9.03 & 46.2 & 9.46 & 46 & 9 & 0.1 & 0 & 0 & 0 \\
\hline Rx_7 & 36.2 & 21.16 & 37 & 21.68 & 37 & 20.5 & 0.3 & 1 & 1 & 1 \\
\hline $\mathrm{Rx} \_8$ & 37.7 & 34.7 & 39.1 & 36.46 & 38 & 34.5 & 0.3 & 0 & 1 & 2 \\
\hline Rx_9 & 34.37 & 34.2 & 34.8 & 35 & 35 & 33.6 & 0.83 & 1 & 0 & 1 \\
\hline $\mathrm{Rx} \_10$ & 22.9 & 33.9 & 23.9 & 37.3 & 23 & 34 & 0.4 & 0 & 1 & 3 \\
\hline $\mathrm{Rx} \_11$ & 23.4 & 41.46 & 23.15 & 40.3 & 24 & 41.5 & 0.6 & 0 & 1 & 1 \\
\hline $\mathrm{Rx} \_12$ & 8.26 & 41.38 & 7.34 & 44.9 & 8 & 41.5 & 0.04 & 0 & 1 & 3 \\
\hline $\mathrm{Rx} \_13$ & 8.59 & 33.55 & 9.3 & 30.7 & 9 & 33.5 & 0.09 & 0 & 1 & 3 \\
\hline $\mathrm{Rx} \_14$ & 8.18 & 13.25 & 9.4 & 20.2 & 8 & 22.7 & 0.22 & 9 & 1 & 3 \\
\hline $\mathrm{Rx} \_15$ & 8.17 & 16.29 & 8.65 & 15.7 & 8 & 16.4 & 0.03 & 0 & 0 & 1 \\
\hline $\mathrm{Rx} \_16$ & 32.4 & 23.4 & 32.26 & 23.2 & 33 & 23.5 & 0.3 & 0 & 0 & 0 \\
\hline $\mathrm{Rx} \_17$ & 30.4 & 13 & 29.45 & 12.7 & 31 & 13.5 & 0.1 & 1 & 1 & 1 \\
\hline $\mathrm{Rx} \_18$ & 14.9 & 25.57 & 14.2 & 32.8 & 15 & 25.5 & 0.2 & 0 & 1 & 7 \\
\hline Rx_19 & 15.04 & 15.2 & 14.8 & 14.3 & 15 & 15 & 0.04 & 0 & 0 & 1 \\
\hline $\mathrm{Rx} \_20$ & 15.15 & 11.7 & 15.01 & 13 & 15 & 11.8 & 0.05 & 0 & 0 & 1 \\
\hline
\end{tabular}

From all previous error, it is clear that both RSS and ToA is effective algorithm to estimate the distance between transmitter and targets. But ToA is slightly better RSS according to the fluctuation of the received signal due to many sources of noise. Like mobile network, wireless local area network and other electric devices. 


\section{Conclusions}

In this paper, a case study of a selected building is simulated using WI software package in order to estimate the locations of many targets. For such an experiment, 20 receivers represent such targets are deployed randomly in the case study area. AoA algorithm is utilized to estimate the target locations which need a distance between the transmitter and target in addition to the angle between an incident wave and reference direction. The distances are estimated in two methods ToA and RSS in order to compare between them to approach accurate estimation. The results confirm that ToA is slightly better than RSS for indoor localization. The resultant errors between actual and estimated locations are in the range of $(0.8,0.6)$ for ToA and the range of $(1.1,7.3)$ for RSS methods.

\section{References}

[1] Chih-Shun Hsu, Yuh-Shyan Chen, Tong-Ying Juang, Yi-Ting Wu.: An Adaptive Wi-Fi Indoor Localization Scheme using Deep Learning . IEEE, (2018)

[2] Shiu Kumar and Seong-Ro Lee.: Localization with RSSI values for Wireless Sensor Networks : An Artificial Neural Network Approach. International Electronic conference on sensors and applications, (2014)

[3] Inam Ullah, Jingyi Che, Xin Su, Christian Esposito, Chang Choi.: Localization and Detection of Targets in Underwater Wireless Sensor Using Distance and Angle Based Algorithms. IEEE, (2019).

[4] Yinan Hou, Yuankai Xue, Cao Chen, Shilin Xiao.:, RSS/AOA based indoor positioning system with a single LED lamp. International Conference on Wireless Communications \& Signal Processing , IEEE, (2015)

[5] Slavisa Tomic, Marko Beko, Rui Dinis , Luís Bernardo.: On Target Localization Using Combined RSS and AoA Measurements. sensors, (2018)

[6] Ayad M. H. Khalel.: Position Location Techniques in Wireless Communication Systems. Electrical Engineering Emphasis on Telecommunications, (2010)

[7] Yanbin Hou, Xiaodong Yang, Qammer H. Abbasi.: Efficient AoA-Based Wireless Indoor Localizationfor Hospital Outpatients Using Mobile Devices. sensors, (2018)

[8] Maan M. Abdulwahid, Oras A. Shareef Al-Ani1, Mahmood F. Mosleh1, Raed A. Abd-Alhmeed.: The Optimum Location for Access Point Deployment based on RSS for Indoor Communication. Journal of Communications, (2019)

[9] R. B. Ertel, P. Cardieri, K. W. Sowerby, T. S. Rappaport, J. H. Reed.: Overview of spatial channel models for antenna array communication systems. IEEE, (1998)

[10] REMCOM Inc.: The Wireless InSite user's manual. version 2.6.3, romcom inc., 315 s. allen st., (2012)

[11] Yanbin Hou, Xiaodong Yang, Qammer H. Abbasi.: Efficient AoA-Based Wireless Indoor Localization for Hospital Outpatients Using Mobile Device. sensors, (2018)

[12] O. Kanhere, T. S. Rappaport.: Position Locationing for Millimeter Wave Systems. Global Communications Conference, Abu Dhabi, U.A.E, pp. 1 (2018)

[13] Sa-e, S.; Chamchoy, M.; promwong, S.: Study on Propagation Path Loss and Performance for Fixed Broadband WiMax. IEEE conferences, (2007).

[14] M. Malajner, K. Benkic, P. Planinsic, and Z. Cucej, "The Accuracy of Propagation Models for Distance Measurement between WSN Nodes", IEEE, International Conference, pp. 1-4, 2009.

[15] Y. S. Cho, J. Kim, W. Y. Yang, C. G. Kang.: MIMO-OFDM Wireless Communications with MATLAB. (2010)

[16] H. Cotuk, K. Bicakci, B. Tavli, E. Uzun.: The Impact of Transmission Power Control Strategies onL of Wireless Sensor Networks. IEEE Transactions on Computers, vol. 63, pp. 2866-2879 (2014)

[17] S. Kim and D.-S. Eom.: Distributed Transmission Power Control for Network Programming in Wireless Sensor Networks. Wireless communications, vol. 72, pp. 1533-1548, (2013) 
[18] L. Cheng, C.-D. Wu, Y.-Z. Zhang.: Indoor Robot Localization Based on Wireless Sensor Networks. IEEE , vol. 57, pp. 1099 1104, (2011)

[19] Salim Latif Mohammed.: Distance Estimation Based on RSSI and Log-Normal Shadowing Models for ZigBee Wireless Sensor Network. Eng. \&Tech.Journal, Vol.34, No.15, (2016)

[20] T. Xiaoyuan, M. Guoqiang, B. D. O. Anderson.: On the Giant Component of Wireless Multihop Networks in the Presence of Shadowing. IEEE Transactions on Vehicular Technology, vol. 58, pp. 5152-5163, (2009)

[21] K. S. Pratap, H.-K. W. Eric, S. Jagruti.: Dual RSSI Trend Based Localization for Wireless Sensor Networks. IEEE Sensors Journal vol. 13, pp.3115 3123, (2013)

[22] Effects of building materials and structures on radio wave propagation above about $100 \mathrm{MHz}$," Recommendation ITU-R P.2040-1, pp. 22-23, (2015) 